\title{
Effect of Dental Thermoplastic Materials on the Clinical Effectiveness of Clear Aligner
}

Hallmann $\mathrm{L}^{1 *}$ and Gerngroß MD $^{2}$

${ }^{1}$ School of Dentistry, Kiel University, Germany

${ }^{2}$ Institute of Material Science, Faculty of Engineering, Kiel University, Germany

*Corresponding author: Lubica Hallmann, School of Dentistry, Kiel University, Arnold-Heller-Str.16, D-24105 Kiel, Germany

Received: February 13, 2021; Accepted: March 05, 2021; Published: March 12, 2021

\section{Abstract}

Objective: The aim of this review is to evaluate the influence of chemical and mechanical material properties properties on the effectiveness of the clinical treatment with a clear aligner.

Methods: A systematic literature study of recent and most relevant publications in this field has been performed to identify the most important material effects on the clinical effectiveness of clear aligners.

Results: Clear aligner devices have found a wide application in orthodontics. The development of new materials which are metal-free and the CAD/CAM engineering technique make it possible to treat the various kinds of malocclusion. Patients prefer clear aligners because of their aesthetics and comfort. The thermoplastic materials have inherent advantages: excellent aesthetic appearance, simple to be used and superior formability. Various thermoplastic materials are being developed for manufacturing of clear aligners. The mechanical properties of polymers play a crucial role in the effectiveness of the therapeutic treatments. Therefore, the elasticity, dimension and environment stability as well as biocompatibility are the main conditions, which the polymer must meet for being used in clear aligners.

Conclusions: The main advantages of clear aligner in comparison to metal or ceramic devices is their excellence aesthetic with better patient acceptance and a general better quality of life. From a clinical point of view, the treatment with clear aligner causes less pain compared to a traditional treatment and also reduce the damage to the gingiva and perodontal tissue. The clinical success of the clear aligner therapy strongly depends on the properties of the used aligner materials.

Significance: The use of thermoplastic materials for orthodontic tooth movements requires a better understanding of the material properties for selecting the optimal material and design.

\section{Introduction}

The increasing aesthetic need of patients for orthodontic devises have lead to the development of materials for aligners [1-11]. Kesling was the first who developed the concept of clear orthodontic devices [11]. In 1997, Align Technology developed Invisalign technique to treat malocclusions, such minor crowding and space closure [10-13]. Clear Aligners (CA) have fond extend application in orthodontics with in the last 15 years through new technologies and new materials to widen the range of tooth movements. The main advantages of $\mathrm{CA}$ are better aesthetic, higher patient acceptance and better life quality. CA treatment results in less pain for patients compared to a traditional fixed treatment [4-12,14-29].

Clear thermoplastic removable aligners are produced by the $\mathrm{CAD} / \mathrm{CAM}$ technology making possible to produce the movements required for a comprehensive orthodontic treatment and more esthetic acceptance in comparision to the traditional stainless steel or aesthethic bracket or wire systems [25,29-32]. The clear aligners production is based on transparent thermoplastic materials offering suitable physical, chemical and mechanical properties intended application $[29,33]$. The mechanical properties of polymers play a crucial role in the effectiveness of the theraupetic treatment. Low stiffness, good defomability, dimensional and environment stability as well as high biocomptability are the conditions that must met by the polymers to be used. The invisible orthodontic force, derived from the deformation and elasticity of aligners, depends on the chemical composition, arrangement and entanglement of the polymer chains, thickness, and the clinical protocol $[1-3,5,17,32-38]$. The ideal clear aligner material must deliver sufficient machanical force to induce an orthodontic movement in a highly controlled manner and simultaneously preventing a damage of the surrounding perodontal Tissues [37].

Polyethylene Terephthalate Glycol (Petg), Polycarbonate (PC) And Thermoplastic Polyurethane (TPU) are mostly used to modify the properties of aligners [5,29,38-39]. PETG, a non-crystalline amorphous co-polymer of Polyethylene Terephthalate (PET), exhibit good mechanical properties, high fatigues resistance, dimensional stability, and solvent resistance [14,18,20,29,33,40-42]. A Glygol Group $(\mathrm{G})$ is added to the backbone of the copolymerizing agent that is a consisting of $31 \% \mathrm{~mol}$ 1,4-cyclohexylenedimethylene terephthalate (PCT) and $69 \mathrm{~mol} \%$ PET [3]. PETG has almost the 
Table 1: Polymers used in this study by Ryokawa [3].

\begin{tabular}{|c|c|c|c|c|}
\hline Code & Thickness $(\mathbf{m m})$ & Product Name & Component \\
\hline Eva & 1 & Bioplast & ethylene-vinyl acetate copolymer \\
\hline PE & 1 & Copyplast & polyethylene & polyethylene terephthalate glycol \\
\hline PETG & 1 & Duran & polypropylene & Sheu-Dental, Gmbh \\
\hline PP & 0.8 & Hardcast & polycarbonate & Sheu-Dental, Gmbh \\
\hline PC & 0.75 & Imprelon « S » & copolyester & Sheu-Dental, Gmbh \\
\hline A+ & 1 & Essix A+ & Raintree Essix, Inc. \\
\hline C+ & 1 & Essix C+ & polypropylene/ethylene copolymer \\
\hline PUR & 0.76 & Invisa lign & polyurethane from methylene diphenyl diisocyanate and 1,6 hexanediol, additive & Align Technology \\
\hline
\end{tabular}

same glass transition temperature (Tg), deformation behavior and optical properties as PET, but does not exhibit the strain-induced crystallization behavior of PET at the production temperature $[10,16,30,39]$.

PC offers an excellent mechanical strength, low water absorption, and transparency making this material very suitable for orthodontic applications [38]. Its properties are very close to the one of Polymethyl Methacrylate (PMMA), but PC offer a higher mechanical strength and is usable in a wider temperature range [33]. PC also has a high transparency in visible light spectrum and provides a higher light transmission behavior than many kinds of glasses $[33,38]$.

Polyuretane (PU) is another elastic polymer that is resistant to abrasion and oil. Its properties strongly depend on the raw materials used for production [43-50]. Thermoplastic Polyurethane (TPU) is one of the most versatile engineering thermoplastics with elastomer properties. TPU exhibit excellent physical properties, chemical resistance, abrasion resistance, adhesion properties and ease of processing [33,51].

The first aligners developed by Align Technology, consisted of a single-layer rigid polyurethane produced from methylene diphenyl diisocyanate and 1,6-hexanediol. To improve its flexibility, fracture resistance, and transparency Smart Track (Align Technology, 2012) developed a new thermoplastic polyurethane [1-2,10,46-48]. The market demand increase of clear aligners has lead to the development of further thermoplastic materials for clear aligners by other companies; such as e. g. Invisaligh, Duran, Biolon, Zendura, Erkodur, Kombiplast, Imprelon, ClearCorrect, Erkoflex 95, Erkoloc pro, etc. [19, 49-50].

Therefore, the aim of this study was to study the influence of materials on the theraupetic treatment with clear aligners.

\section{Material Properties of Aligners \\ Water absorption}

The mechanical properties of dental polymers show a multitude of influence factors, such as structural factors (molecular and crystal structures etc.) and enviromental factors, (temperature, humidity etc.) $[3,29]$. The employed polymers are typically either amorphous or semi-crystalline. Amorphous polymers usually appear clear because visible light can pass through, while many semi-crystalline and crystalline polymers are opaque because they consist of mixture of crystalline and amorphous phases with different refractive indexes $[3,29]$. The crystalline phase in semi-crystalline polymers can serve as a reinforcement gridleading to an improvement in the mechanical properties over a wide range of applications. Low crystallinity of polymers typically means: high flexibility, high elasticity, welladaptable to the tooth morphology, but on the other hand they providing low tensile strength, low chemical resistance and stability [29]. From a clinical point of view, polymers with high flexibility and elasticity are more comfortable for patients to insert or remove the aligners. Furthermore, they adapt better to the complexity of the tooth morphology, adhere perfectly to any surface. Compared to aligners of rigid materials they also ensure a continuity of force expression during the orthodontic treatment [29].

Ryokawa et al. investigated the properties of dental thermoplastic materials for clear aligner [3]. The polymeric materials for orthodontic applications used in this study are listed in (Table 1).

Figure 1 displays the time-dependent water absorption behavior of the investigated polymers. Depending on their water absorption behavior the investigated polymers can be divided into two groups. The group 1 polymers include EVA, PETG, PC, $\mathrm{A}^{+}$, and PUR. These polymers show a steep increase of water absorption within the first 24 hours of water exposure before approaching a saturation level. This saturation level can strongly vary depending on the respective polymer.

The group 2 polymers include $\mathrm{PE}, \mathrm{PP}$, and $\mathrm{C}^{+}$. They exhibit a rather small increase of water absorption within $72 \mathrm{~h}$ of water exposure before reaching their corresponding saturation limit.

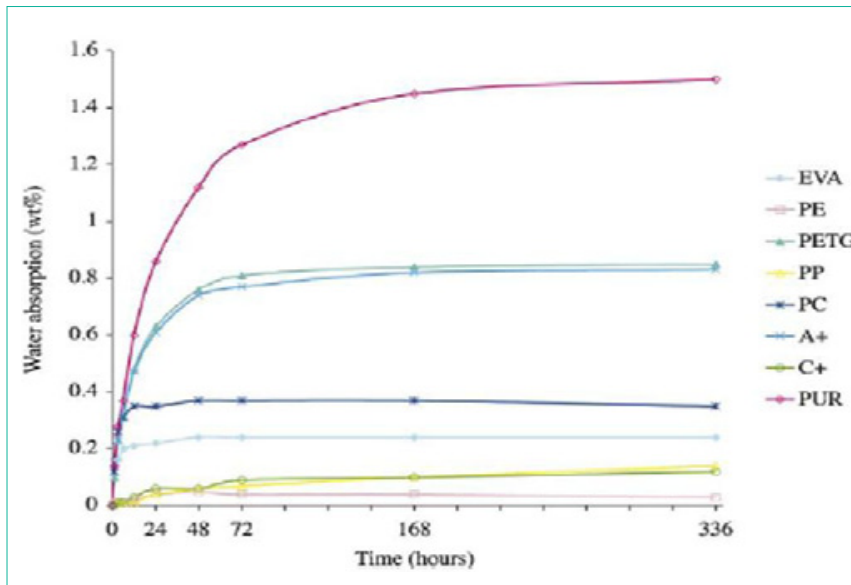

Figure 1: Time-dependent water absorption behavior (up to 2 weeks) of eight thermoplastic polymers [3] 


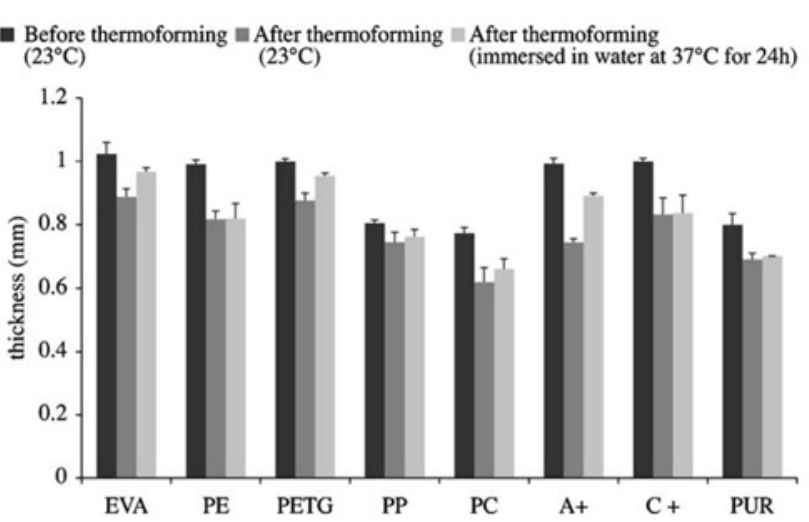

Figure 2: Thickness change in under the three conditions [3]

In this study PUR showed the highest water absorption of approximately $1.5 \mathrm{wt} \%$ (Figure 1), followed by PETG and $\mathrm{A}^{+}$. PE had the lower water absorption after two weeks, with about $0.03 \mathrm{wt} \%$. So, in absolute numbers, the water uptake of group 2 polymers is only a fraction of the one of group 1 polymers.

Typically the water absorption behavior of polymers is influenced by several factors. The major factors among them there are : 1) the interaction of water molecule with hydrophilic/polar groups of polymer (hydrogen bonding) and 2) the level of free volume depending on the polymer structure (amorphous, crystalline). As a result, crystalline polymers with high molecular densities show a lower rate of water absorption, while amorphous polymers show higher rates of water absorption due to the drastically larger free volume [3].

Furthermore Ryokawa et al. also investigated the effect of thermoforming and thermoforming with subsequent water exposure on the thickness of the polymer sheet. This subsequent water absorption of the polymer is of high importance because it is can result in intraoral dimension changes leading to changes in the orthodontic forces of the aligner [3,42].

As can be seen from Figure 2, all investigated polymers exhibit a thickness reduction after thermoforming. A subsequent water exposure for 24 hours results in a mixed behavior among investigated polymers with $\mathrm{PE}$ showing the smallest linear expansion rate followed by C+, PUR, PP, PV, PETG, EVA and A+. Taking this into account and the time-dependent water absorption behavior of different polymers one can be concluded that the thermoforming process plays an important role for the application of clear aligners. To improve the mechanical properties of clear aligner Ahn et al. produced a threelayers clear aligner. The first layer is composed of a reinforced resin core, the middle layer of a soft-type of Thermoplastic Polyurethane (TPU), and the outer layer of a hard-type thermoplastic material (PETG).

Figure 3 presents the time-dependent and layer number dependent water absorption behavior. The time-dependence is very similar to the one shown by Ryokawa et al. in [3]. The lowest water absorption with $0.77 \mathrm{wt} \%$ after 336 hours is observed for the singlelayer specimens, while the double-layer specimens show the highest water absorption. Adding a third layer results in a water uptake

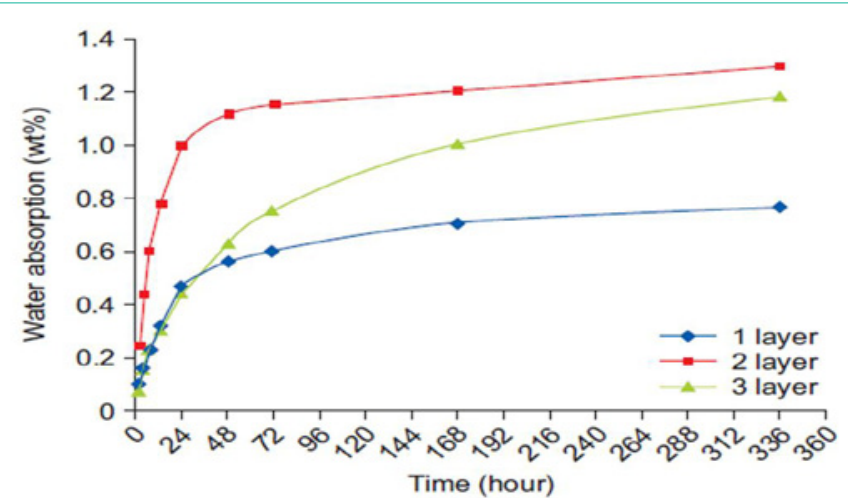

Figure 3: Time-dependent water absorption (up to 2 weeks) depending on layer number of the aligner [42]

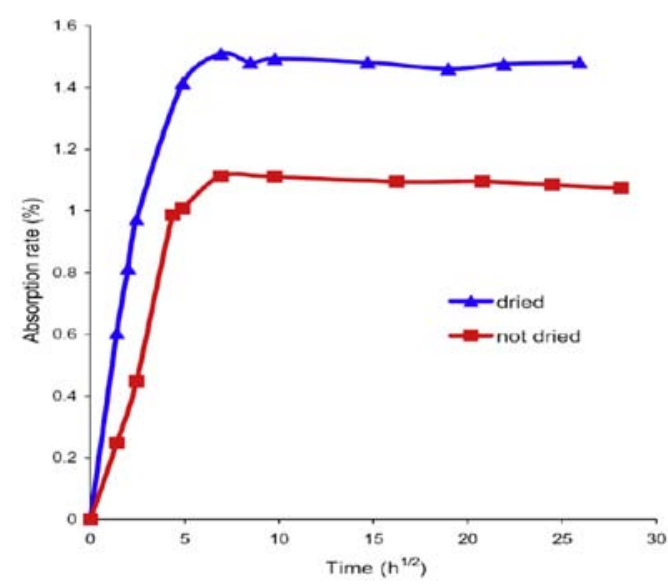

Figure 4: Water absorption rate of TPU for dried and not-dried specimens [44].

between both arrangements. One can understand this finding in the way that the PETG as third layer is shielding the underlying TPU from water uptake, so that the overall water uptake of the three-layer stack is reduced compared to the double-layer stack. This shielding ability is especially important taking into account that water-induced polymer swelling after intraoral exposure can causes dimensional change of the aligner. This could also lead to irreversible degradation of the polymer backbone chain by hydrolysis [42].

Boubakri et al. studied the kinetics of water diffusion into TPU. They investigated the effect of drying on the water absorption rate. They formed two groups:

- $\quad$ The specimens are dried at temperature of $70^{\circ} \mathrm{C}$ for $48 \mathrm{~h}$

- The specimens were not dried.

Figure 4 depicts the water absorption rate of the dried and not dried TPU specimens.

One can clearly see that the dried TPU specimens exhibit a steeper increasing slope of the water absorption rate compared to the not-dried ones. In addition to that the saturation level of the dried TPU specimens is significantly higher compared to not-dried ones.

These authors assume that the time to reach the saturation level 


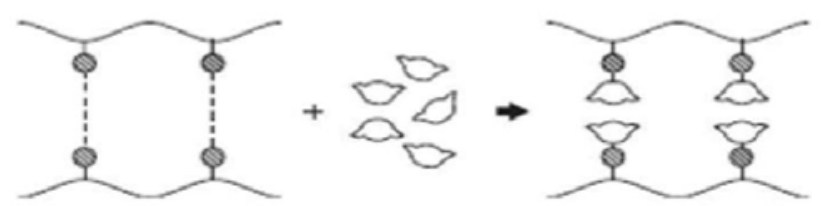

Polymer + water polymer plasticized by water

Figure 5: Destruction of intramolecular bonds between polymer chains by water molecule diffusion [44]

Table 2: Fluid absorption after storage in artificial saliva [52]

\begin{tabular}{|c|c|}
\hline Material & Fluid absorption (\%wt) \\
\hline Duran & 0.375 \\
\hline Biolon & 0.438 \\
\hline Zendura & 0.719 \\
\hline
\end{tabular}

depends on the diffusion kinetics, the layers thickness and on the temperature (Figure 4). These results are in good agreement with the results of other research studies about polyurethane $[3,42]$.

TPU has two functional groups $(\mathrm{C}=\mathrm{O}$ and $\mathrm{N}-\mathrm{H})$ that form intermolecular hydrogen bonds that are responsible for the material cohesion. The absorption of water molecules destroys these intramolecular bonds between the chains. This leads to a decrease in the mechanical properties such as tensile strength, Young's modulus, wear resistance and an increase in molecular mobility. The increase of molecular mobility is responsible for the decrease in the glass transition Temperature $(\mathrm{Tg})$ and the reorganization of the polymer chain structure. This results in an increase in free volume, schematically shown in (Figure 5) [44]. The absorption of water molecule does not change the chemistry of the polymers, but destroy the hydrogen bond between $\mathrm{C}=\mathrm{O}$ and $\mathrm{N}-\mathrm{H}$ groups having a negative effect on the mechanical properties $[3,18,20,51]$.

Tamburino et al. studied the properties of materials for the thermoforming production of aligners. The materials used in their study were: Duran (PETG, Sheu dental GmbH), Biolon (PETG, Dreve Dentamid $\mathrm{GmbH}$ ) and Zendura (PU, Zendura Dental). Artificial saliva was used as an aging agent at a temperature of $37^{\circ} \mathrm{C}$ for 7 days [52]. The water absorption of these tested materials is presented in (Table 2).

As can be seen from (Table 2), the fluid uptake of Duran material is only almost half of the Zendura one. In addition to higher water uptake the authors found a deterioration of the mechanical properties of the Zendura that can be explained by the previously described mechanism of intramolecular bond destruction by water molecules [52].

Zhang et al. studied the properties of three aligner materials (table 3 ). They mixed the polymers in various combinations and ratios before performing the thermoforming process generating PETG/PC/ TPU composites. As control groups they used Erkodur (PETG) and Biolon (PETG).

The lowest water absorption after 336 hours was achieved for a PETG/PC/TPU blending ratio of $80 / 10 / 10(0.52 \mathrm{wt} \%)$, which is significantly lower compared to the control group of Erkodur (0.80

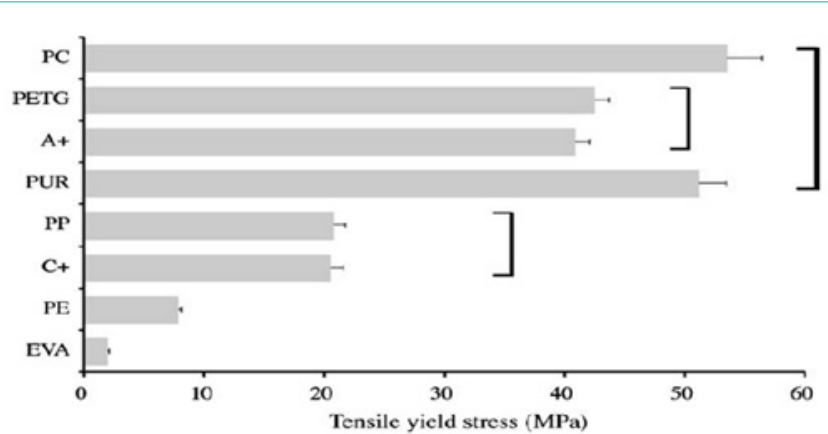

Figure 6: Comparison of tensile yield stress of different polymers for aligners in simulated intraoral environment [3].

wt\%) and Biolon (0.81 wt\%). Similiar results were also obtained for the blending ration $70 / 10 / 20(0.57 \mathrm{wt} \%)$.

These authors assume that the water absorption of PETG/PC/ TPU increases with an increase in TPU blending ratio due to the polar group [33].

Water absorption can have negative effects on the mechanical properties of polymers leading to irreversible degradation, because water uptake is often accompanied by swelling and thus, a degradation of the polymers $[33,44]$. Besides the degradation effect, the swelling also leads to dimensional changes of the mouth devices, which influences the orthodontic forces them. Therefore, an ideal thermoplastic material for clear aligner should have a low water absorption [33,44].

\section{Mechanical properties}

According to Ryokawa et al. the tensile yield stress of the aligners depends on the chemical composition of the polymers as depicted in (Figure 6) [3].

As can be seen from Fig. 6 the tensile yield stress of the investigated polymers degreases as following: $\mathrm{PC} \geq \mathrm{PUR}>\mathrm{PETG} \geq \mathrm{A}+>\mathrm{PP} \geq \mathrm{C}+>$ $\mathrm{PE} \geq$ EVA. This result can be understood in term of the presence respectively the degree of crystallinity of the corresponding polymers. PC, PETG, $\mathrm{A}^{+}, \mathrm{PUR}$ as amorphous polymers show a higher tensile yield stress than PP, $\mathrm{C}^{+}, \mathrm{PE}$ and EVA which are semi-crystalline. Besides this structural influence on the mechanical propertries, also the glass transition temperature ( $\mathrm{Tg}$ ) plays an important role. Below $\mathrm{Tg}$ amorphous and semi-crystalline polymers behave very similar. Their mechanical behavior is typically rigid and glassy. Above $\mathrm{Tg}$ the behavior of amorphous and semi-crystalline polymers partially deviates. Amorphous polymers soften and can become flexible elastomers or even a viscous liquid [3]. Semi-crystalline polymers also soften and can become flexible depending strongly on the degree of crystallinity [3]. Typically, well above Tg the crystalline phase of the semi-crystalline polymer can also vanish due to melting.

This means, the mechanical properties of polymers with $\mathrm{Tg}$ around room temperature may be influenced tremendously by changes in temperature [3]. Another factor affecting the properties of the polymers is the molecular orientantion of the polymer chains. From the results of the study of Ryokawa et al. it can be concluded that the mechanical properties depend on a multitude of factors, starting from the phase state (crystalline or amorphous) of the polymers, their 


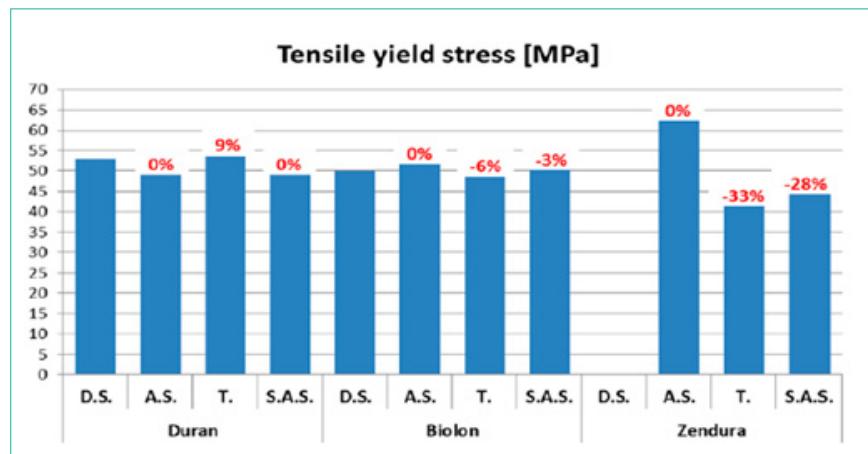

Figure 7: Mean value of tensile yield stress for each materials and test condition (DS: Data Sheet; AS: As Supplied; T: Thermoformed; SAS: Stored in Artificial Saliva) [52].

water absorption ability, their molecular orientation and processing history, as well as their application conditions $[3,18,33,41,44]$.

Ahn et al. investigated the tensile strength of three layers clear aligner in various layer stacks at room temperature, but not the effect of water absorption on the tensile strength. The highest tensile strength is obtained for a three-layer clear aligner $(469 \mathrm{~N})$ followed from double-layer $(317 \mathrm{~N})$ and single-layer $(258 \mathrm{~N})$. Based on this study it seems that the three-layer clear aligner shows the best mechanical performance, but the lack of aging results make it is difficult to evaluate the positive effect of three-layer clear aligner on the mechanical properties of this new design.

Condo et al. investigated the effect of additive on the mechanical properties of two generations of teeth aligners in-vitro tests. For their study they used two polymers: EX30 and LD30. Both materials are polyurethane-based and have almost the same chemical composition. Both materials are considered semi-crystalline materials. The difference between both materials is the second compound in the LD30. EX30 exhibited higher crystallinity (37\%) than LD30 (28\%). LD30 is more translucent, more flexible and more elastic than EX30. After oral aging, both materials showed an increase in crystallinity. The mechanical properties of EX 30 and LD30 changed after oral usage. They became harder and more hyperplastic which is uncomfortable for the patients [29]. The authors emphasize that the quality of the orthodontic force exerted by aligner depends on the mechanical properties in the manufacturing process of materials itself [29]. From clinical application's point view, the orthodontic devices are subjected to both short- (occurs on inserting or removing the aligner) and long-term load (occurs at night as a result of chewing force) [29]. Although there is long-term stress in the oral cavity, a thermoplastic orthodontic device must, however exercise continuous and controlled orthodontic forces, like those that are supposed to cause tooth movement $[29,53,47]$. Therefore, these authors concluded that LD30 material meet aligner requirements better than EX30 material [29].

Brandely et al. [18], Papadopoulouet al. [46], Schuster et al. [50] examined the mechanical properties of the Invisalign aligner after intra-oral aging. According to these authors different factors might be responsible for the deterioration of the aligner's mechanical properties after oral use. The first can be attributed to the material itself. Polyurethane-based materials are made up of hard and soft segments. The hard segment, a biphenyl structure, provides stability and sufficient reactivity to form a byproduct-free polymer [50]. The soft segments tend to be oriented perpendicular to the applied stress direction causing them to fracture into smaller segments and thus, enabling further deformation. A second factor could be the leaching of matrix plasticizers causing a reduction of residual stresses from the manufacturing process. Another factor could be attributed to the fact that during the intraoral use the material is exposed to mechanical abrasion generated during masticating movement, along with chemical abrasion from the consumption of acidic beverages and the action of enzymes. Human saliva contains a complex of minerals, lipids, proteins and has a fluctuating $\mathrm{pH}$, which may also affect the mechanical properties of polymers. [46,50]. Papadopulou et al. concluded that the use of these devices is not recommended for more than two weeks due to the intra-oral aging effect on the above described properties [46].

Tamburino et al. investigated the mechanical properties of the aligner materials Duran, Biolon and Zendura in the as delivered state, after thermoforming and after storage in artificial saliva [52].

The results for the tensile yield stress measurements are depicted in Figure 7. The authors found that the tensile yield stress of the Duran and Biolon materials only slightly changed after thermoforming ( $9 \%$ increase for Duran, 6\% decrease for Biolon), while is decrease by one third for the Zendura.

After exposure to artificial saliva, the tensile yield stress of the Duran material decrease back to it as-supplied strength, while the tensile yield stress of Biolon and Zendura materials slightly increase (to $-3 \%$ respectively to $-28 \%$ ). Based on their finding these authors propose to select a material for orthodontic devices after characterizing its mechanical properties after the corresponding manufacturing process and storage test in an intraoral simulation environment [52].

Zhang et al. investigated the mechanical properties of PETG, PC, and TPU in various blending ratios. As control group they used Erkodur and Biolon materials. The aim of their study was to improve the mechanical properties of PETG because the tear strength (42.12 $\mathrm{MPa})$ and elongation-at-break value (141.22\%) were lower than Erlodur and Biolon [33]. These authors achieved optimal results for a PETG/PC/TPU blending ration of 70/10/20: tear strength is about $50.23 \mathrm{MPa}$ with an elongation-at-break of about $155.99 \%$ and a stress relaxation rate $0.0136 \mathrm{~N} / \mathrm{s}$ after 1 hour. The authors concluded that $\mathrm{PETG} / \mathrm{PC} / \mathrm{TPU}$ at a properly blending ration could provide sufficient and more sustainable orthodontic forces during oral treatment than Erkodur and Biolon [33].

\section{Elastic modulus}

A higher elastic modulus is benefical for aligners as it increases the force delivery capacity of the aligner under constant strain [10]. In addition, materials with a higher elastic modulus can provide the same forces from thinner dimensions [10].

Ryokawa et al. calculated the elastic modulus from the measured elastic stress and strain. The elastic modulus is proportional to the material stiffness. According to the authors, EVA and PE exhibit the lowest elastic modulus and tensile yield stress. In addition they also have a lower Tg and thus, are more strongly affected by themperature changes during oral application. These materials show a significant 


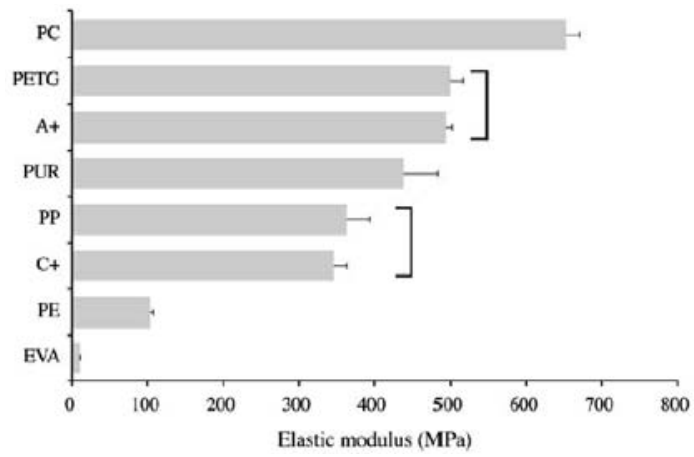

Figure 8: Comparison of elastic moduli among materials in a simulated intraoral environment [3].

Table 3: Polymers used for clear aligner [33].

\begin{tabular}{|c|c|c|}
\hline Materials & Molecular Weight & Company \\
\hline PETG & 26000 & Eastman Chemical Co. Tennessee, USA \\
\hline PC & 23500 & Unitika, Osaka, Japan \\
\hline TPU & 18000 & Bayer, Leverkusen, Germany \\
\hline
\end{tabular}

decrease in their mechanical properties after oral use (Figures 6,8) [3]. The decrease in elastic modulus is due to an increase in temperature and water absorbtion. It is accompanied by the decrease in the initial forces that the aligner applies in the early phase after intraoral use [34].

In their study [52], Tamburino et al. also investigated the elastic modulus of the aligner materials Duran, Biolon and Zendura in the as delivered state, after thermoforming and after storage in artificial saliva.

As can be seen from (Figure 9), the elastic modulus of the Duran and Zendura materials increased by $11 \%$ respectively $17 \%$ after thermoforming, while the one of the Biolon material falls by $7 \%$.

Looking at the elastic modulus after artificial saliva exposure of the materials shows a different behavior. The elastic modulus of Bicolon and Zendura material is rather stable, while a significant decrease was observed for Duran [52]. This decrease can be explained by water absorption occurring during the storage in artificail saliva fluid. The absorption of water can act as a plastizier, which leads to a Tg reduction, especially for amorphous polymers. It is known for these polymers that their elastic modulus exhibit a linear temperature dependence, near their corresponding Tg the elastic modulus reduces [51,54].

\section{Influence of Thermoforming}

The thermoforming process affects the mechanical properties of clear aligners $[3,10,53]$. After thermoforming a reduction in the tensile yield stress and elastic modulus of the investigated polymers is found.

The authors explain this behavior in terms of a decrease in molecular weight due to the heat-treatment, the molecular orientation, and the rapid cooling preventing further crystallization of the polymers and generating residual stresses. $[3,10]$.

Ruy et al. investigated the effect of thermoforming on the physical

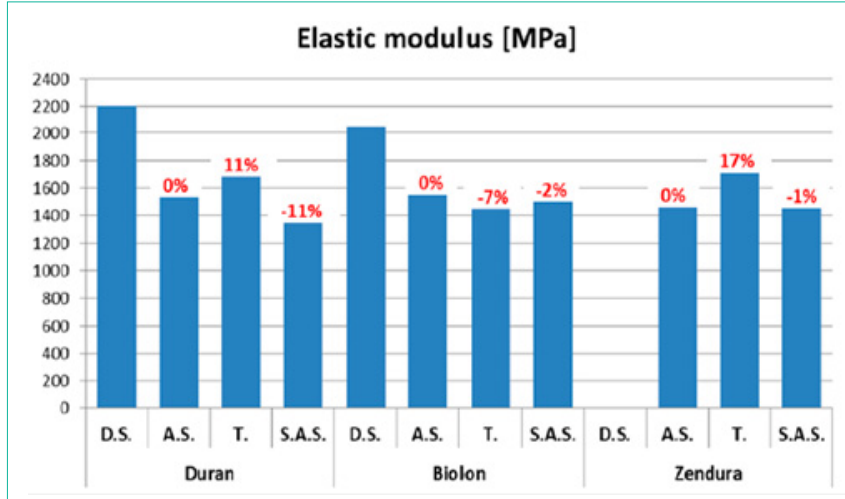

Figure 9: Mean value of elastic modulus for each materials and test condition (DS: Data Sheet; AS: As Supplied; T: Thermoformed; SAS: Stored in Artificial Saliva) [52].

and mechanical properties of materials for clear aligners. They observed that the optical transparenty, the tensile force and elastic modulus of the aligner materials decrease after the thermoforming process, while water absorption was increased. The materials subjected to a temperature above their corresponding Tg can easy deform and decrease in thickness. The crystallinity of thermoplastic materials is changed after thermoforming accounting for the change in the mechanical properties. Thus these authors cocluded that the properties of materials such transparence, water absorption and solubility, surface hardness, flexure and elastic moduli, and the tensile and flexural forces can alter significanty after thermoforming [31]. Furthermore, they recommanded to evaluate the durabilty of these materials after thermoforming in order to characterize their properties for their clinical application [31]. From the clinical point of view, the authors also suggested choosing the polymers depending on the treatment required, as some of them show a significant decrease in flexural strength after thermoforming and exhibit permanent deformation during treatment. On the other hand, the application of large forces to the teeth can lead to absorption of the apical root [31].

\section{Future}

The dental industry developed a 3D-printing method for the production of clear aligners in order to reduce the negative impact of thermoforming on the properties of clear aligners. In the future, the properties of these new clear aligner types will be investigated.

\section{Conclusion}

Clear aligner are used in orthodontic to treat mild to complex dental malocclusions. They can be used by orthodontist and general dentists. The main advantages of clear aligner in comparison to metal or ceramic devices is their excellence aesthetic with better patient acceptance and a general better quality of life. From a clinical point of view, the treatment with clear aligner causes less pain compared to a traditional treatment and also reduce the damage to the gingiva and perodontal tissue. The clinical success of the clear aligner therapy strongly depends on the properties of the used aligner materials.

Here, especially the mechanical and chemical properties of the used polymers play a crucial role in the effectiveness of theraupetic treatmens because the invisible orthodontic forces is derived from the deformation and elasticity of the aligner. Thus, the elasticity, 
dimension and environment stability as well as biocompatibility, are of the major importance depends. These materila properties can be further ascribed to the chemical composition of the polymers, the arrangement and entanglement of the polymer chains, the layer thickness and the clinical protocols.

\section{References}

1. Lombardo L, Martines E, Mazzanti V, Arreghini A, Mollica F, Siciliani G. Stress relaxation properties of four orthodontic aligner materials : A 24-hour in vitro study. Angle Orthodontist. 2017; 87: 11-18.

2. Lombardo L, Palone M, Longo M, Arveda N, Nacuchi M, Pascalis FD, et al. MicroCT X-ray comparison of aligner gap and thickness of six brands of aligners: an in-vitro study. Progress in Orthodontics. 2020; 21: 12.

3. Ryokawa H, Miyazaki Y, Fujishima A, Miyazaki T, Maki K. The mechanical properties of dental thermoplastic materials in a simulated intraora environment. Orthodontic Waves. 2006; 65: 64-71.

4. Weir T. Clear aligner in orthodontic treatment. Australian Dental Journal. 2017; 62: 58-62.

5. Liu C-L, Sun W-T, Liao W, Lu W-X. Li Q-W, Jeong Y, et al. Colour stabilities of three types of orthodontic clear aligners exposed to staining agents. International Journal Oral Science. 2016; 8: 246-253.

6. Inoue S, Yamaguchi S, Uyama H, Yamashiro T, Imazato S. Orthodontic aligner incorporating eucommia ulmoides exert low continuous force: in vitro study. Materials. 2020; 13: 4085.

7. Buschang PH, Ross M, Shaw SG, Crosby D, Campbell PM. Predicted and actual end-of-treatment occlusion produced with aligner therapy. Angle Orthodontist. 2015; 85: 723-727.

8. Barone S, Paoli A, Razionale AV, Savignano R. Computational design and engineering of polymeric orthodontic aligners. International Journal for Numerical Methods in Biomedical Engineering. 2017; 33: e2839.

9. Dasy H, Dasy a, Asatrian G, Rozsa N, Lee H-F, Kwak JH. Effect of variable attachment shapes and aligner material on aligner retention. Angle Orthodontist. 2015; 85: 934-940.

10. Alexandropoulos A, Jabbari YSA, Zinelis S, Eliades T. Chemical and mechanical characteristics of contemporary thermoplastic orthodontic materials. Australian Orthodontic Journal. 2015; 31: 165-170.

11. Kesling HD. Coordinating the predetermined pattern and tooth positioner with conventional treatment. American Journal Orthodontics and Oral Surgery. 1946: 32; 285-293.

12. Rossini G, Parrini S, Castroflorio T, Deregibus A, Debernardi CL. Efficasy of clear aligners in controlling orthodontic tooth movement A systematic review. Angle Orthodontist Journal. 2015; 85: 881-889.

13. d'Apuzzo F, Perillo L, Carrico CK, Castroflorio T, Grassia V, Lindauer SJ, et al. Clear aligner treatment : different perprectices between orthodontists and general dentists. Progress in Orthodontic. 2019; 20: 10.

14. Bernard G, Rompre P, Tavares JR, Monpetit A. Colorimetric and spectrophotometric measurements of orthodontic thermoplastic aligners exposed to various staining sources and cleaning methods. Head \& Face Medicine. 2020;16: 2

15. Ma YS, Fang DY, Zang N, Ding XJ, Zhang KY, Bai YX. Mechanical properties of orthodontic thermoplastic PETG PC2858 after blending. Chinese Journal Dental Research. 2016; 19: 43-48.

16. Hennesy J, Al-Awadhi EA. Clear aligners generations and orthodontic tooth movement. Journal of Orthodontics. 2016; 43: 68-76.

17. Shalish M, Kazaz-Copper R, Ivgi I, Canetti L, Tsur B, Bachar E, et al. Adult patients' adjustabilty to orthodontic appliances. Part I: a comparision between Labial, Lingual, and Invisalign ${ }^{\mathrm{TM}}$. European Journal of Orthodontics. 2012 34: 724-730.

18. Brandley TG, Teske L, Eliade G, Zinelis S, Eliades T. Do the mechanical and chemical properties of Invisalign ${ }^{\mathrm{TM}}$ appliances change after use? A retrivial analysis. European Journal of Orthodontics. 2016; 38: 27-31.
19. Papadimitriou A, Mousoulea S, Gkantidis N, Kloukos D. Clinical effectiveness of Invisaligh orthodontic treatments: a systematic review. Progress in Orthodontics 2018; 19: 37.

20. Liu C-L, Sun W-T, Liao W, Lu W-X, Li Q-W, Jeong Y, et al. Colour stability of three types of orhodontic clear aligners exposed to staining agents. International Journal of Oral Sciences. 2016; 8: 246-253.

21. Fang $D$, Zhang $N$, Chen $H$, Bai $Y$. Dynamic stress relaxation of orthodontic thermoplastic materials in a stimulated oral environment. Dental Materials Journal. 2013; 32: 946-951.

22. Elkholy F, Schmidt S, Amirkhani M, Schmidt F, Lapatki BG. Mechanical characterization of thermoplastic aligner materials : recomandations for test parameter standartization. Journal of Healthcare Engineering. 2019.

23. Ercoli F, Tepedino M, Parziale V, Luzi C. A comparative study of two different clear aligner systems. Progress in Orthodontics. 2014; 15: 31.

24. Fujiyama K, Honjo T, Suzuki M, Matsuoka S, Deguci T. Analysis of pain level in cases treated with invisalign aligner: comparison with fixed edgewise appliance therapy. Progress in Orthodontics. 2014; 15: 64.

25. Iliadi A, Koletsi D, Papageorgiou SN, Eliades T. Safety considerations for thermoplastic-type appliances used as orthodontic aligners or retainers. A systematic review and meta-analysis of clinical and in-vitro research. Materials. 2020; 13: 1843

26. Inoue S, Yamaguchi S, Uyama H, Yamashiro T, Imazato S. Orthodontic aligner incorporating eucommia ulmoides exerts low contnuous force: in vitro study. Materials. 2020; 13: 4085.

27. Melkos AB. Advances in digital technology and orthodontics: a reference to the Invisalign method. Medical Science Monitor. 2005; 11: 39-42.

28. Zheng M, Liu R, Ni Z, Yu Z. Efficiency, effectiveness and treatment stability of clear aligners: A systematic review and meta-analysis. Orthodontics \& Craniofacial Research. 2017; 20: 127-133.

29. Condo R, Pazzini L, Cerroni L, Pasquantonio G, Lagana G, Pecora A, et al. Mechanical properties of two generations of teeth aligners: change analysis during oral permanence. Dental Material Journal. 2018; 37: 835-842.

30. Abdallah M-N, Lou T, retrouvey J-M, Suri S. Biomaterials used in orthodontics: brackets, archwires, aand clear aligner. Advanced Dental Biomaterials. 2019; 541-579.

31. Ryu J-H, Kwon J-S, Jiang HB, Cha J-Y, Kim K-M. Effect of thermoforming on the physical and mechanical properties of thermoplastic materials for transparent orthodontic aligners. Korean Journal Orthodontics. 2018; 48: 316-325

32. Key, Zhu Y, Zhu M. A comparison of treatment effectiveness between clear aligner and fixed appliance therapies. BMC Oral Health. 2019; 19: 24.

33. Zhang N, Bai $Y$, Ding $X$, Zhang $Y$. Preparation and characterization of thermoplastic materials for invisible orthodontic. Dental Materials Journal. 2011; 30: 954-059

34. Ihlsen BA, Willmann JH, Nimer A, Drescher D. Effect of in vitro aging by water immersion and thermocycling on the mechanical properties of PETG aligner materia. Journal Orofacial Orthopedics. 2019; 80: 292-303.

35. Hahn W, Engelke B, Jung K, Dathe H, Kramer FJ, Rodig T, et al. The influence of occlusal forces on force delivery properties of aligners during rotation of an upper central incisor. Angle Orthotontist. 2011; 81: 1057-1063.

36. Kohda N, Lijima M, Muguruma T, Brantley WA, Ahluwalia KS, Mizuguchi I. Effect of mechanical properties of thermoplastic materials on the initial force of thermoplastic appliance. Angle Orthodontist. 2013; 83: 476-483.

37. Fujiyama K, Honjo T, Suzuki M, Matsuoka S, Deguchi T. Analysis of paim level in cases treated with Insisaligh aligner : comparison with fixed edgewise appliance therapy. Progress in Orthodontics. 2014; 15: 64.

38. LeGrand DG, Bendler JT. Handbook of Polycarbonate Science and Technology. Marcel Dekker Inc., New York. 1999; 33-41.

39. Lu QW, Macosko CW. Comparing the compatibility of various functionalized polypropylenes with Thermoplastic Polyurethane (TPU). Polymer. 2004; 45: 1981-1991. 
40. Dupaix RB, Boyce MC. Finite strain behaviorof Polyethylene Terephtalate (PET) and Polyethylene Terephtalate-Glycol (PETG). Polymer. 2005; 46: 4827-4838.

41. Ahn H-W, Ha H-R, Lim H-N, Choi S. Effect of aging procedures on the molecular, biochemical, morphological, and mechanical properties of vacuum-formed retainers. Journal Mechanical Behavior Biomedical of Materials. 2015; 51: 356-366.

42. Ahn H-W, Kim KA, Lim S-H. A new type of clear orthodontic retaine incorporating multi-layer hybrid materials. Korean Journal Orthodontics. 2015; 45: 268-272

43. Martin DJ, Osman AF, Andriani Y, Edwards GA. Thermoplastic Polyurethane (TPU)-based polymer nanocomposites. Advances in Polyme Nanocomposites. 2012; 321-350.

44. Boubacri A, Elleuch K, Guermazi N, Ayedi HF. Investigations on hygrothermal aging of thermoplastic polyurethaane material. Materials and Design. 2009 30: 3958-3965.

45. Russo P, Lavorgna M, Piscitelli F, Acierno D, Maio LD. Thermoplastic polyurethane films reinforced with carbon nanotubes: the effect of processing on the structure and mechanical properties. European Polymer Journal. 2013; 49: 379-388

46. Papadopoulou AK, Cantele A, Polychronis G, Zinelis S, Eliades T. Changes in roughness and mechanical properties of Invisalign ${ }^{\circledR}$ appliances after oneand two-weeks use. Materials. 2019; 12: 2406.
47. Jaggy F, Zinelis S, Polychronis G, Patcas R, Schatzle M, Eliaddes G, et al. ATR-FTIR analysis and one-week stress relaxation of four orthodontic aligner materials. Materials. 2020; 13: 1868.

48. Kwon J-S, Lee Y-K, Lim B-S, Lim Y-K. Force delivery properties of thermoplastic orthodontic materials. American Journal Orthodontics and Dentofacial Orthopedics. 2008; 133: 228-234.

49. Martina S, Rongo R, Bucci R, Razionale AV, Valletta R, D' Anto. In vitro cytoxicity of different thermoplastic materials for clear aligners. Angle Orthodontist. 2019; 89: 942-945

50. Schuster S, Eliades G, Zinelis S, Eliades T, Bradley G. Structural conformation and leaching from in vitro aged and retrieved Invisalign appliances. American Journal Orthodontics and Dentofacial Orthopedics. 2004; 126: 725-728.

51. Yang B, Huang W, Li C, Li L. Effect of moisture on the thermomechanical properties of a polyurethane shape memory polymer. Polymer. 2006; 47: 1348-1356.

52. Tamburrino $F$, D'Anto $V$, Bucci $R$, Alessandri-Bonett $G$, Barone $S$ Razionale AV. Mechanical properties of thermoplastic polymers for aligner manufacturing: in vitro study. Dentistry Journal. 2020; 8: 47.

53. Bucci R, Rongo R, Levate C, Michelotti A, Barone S, Razionale AV, et al. Thickness of orthodontic clear aligners after thermoforming and after 10 days of intraoral exposure: a prospective clinical study. Progress in Orthodontics. 2019; 20: 36 